\title{
Identifying next relevant variables for segmentation by using feature selection approaches
}

\author{
Alex Seret $^{\mathrm{a}, *}$, Sebastian Maldonado ${ }^{\mathrm{b}}$, Bart Baesens $^{\mathrm{a}, \mathrm{c}, \mathrm{d}}$ \\ ${ }^{a}$ Department of Decision Sciences and Information Management, KU Leuven, Naamsestraat 69, B-3000 Leuven, Belgium \\ ${ }^{b}$ Faculty of Engineering and Applied Sciences, Universidad de los Andes, Las Condes, Santiago, Chile \\ ${ }^{c}$ School of Management, University of Southampton, Highfield Southampton, SO17 1BJ, United Kingdom \\ ${ }^{d}$ Vlerick, Leuven-Gent Management School, Reep 1, B-9000 Gent, Belgium
}

\begin{abstract}
Data mining techniques are widely used by researchers and companies in order to solve problems in a myriad of domains. While these techniques are being adopted and used in daily activities, new operational challenges are encountered concerning the steps following this adoption. In this paper, the problem of updating and improving an existing clustering model by adding relevant new variables is studied. A relevant variable is here defined as a feature which is highly correlated with the current structure of the data, since our main goal is to improve the model by adding new information to the current segmentation, but without modifying it significantly. For this purpose, a general framework is proposed, and subsequently applied in a real business context involving an event organizer facing this problem. Based on extensive experiments based on real data, the performance of the proposed approach is compared to existing methods using different evaluation metrics, leading to the conclusion that the proposed technique is performing better for this specific problem.
\end{abstract}

Keywords: Clustering, Feature selection, Filter methods, Customer segmentation, Business analytics

\section{Introduction}

Using data mining techniques as a support for operational decisions seems to be present in the agenda of more and more companies willing to monetize their data. While some companies are only starting the journey, some others are already a step further, facing operational challenges related to the post-processing and updating of the generated knowledge. As a well-known and hence frequent data mining application, the segmentation of customers using clustering techniques is also impacted by these considerations (Baesens, 2014). Once segments are obtained, different practical steps can be considered. If the clustering structure based on which the segmentation is made is taken as a fixed structure and if some characteristics of the customers can vary trough time, a logical next step could be to update the positions of the customers, hence updating their memberships to the segments. By capturing these movements relatively to the fixed structure, the dynamics of the customers can thus be explored (see e.g. Seret et al., 2014a). On the other hand, one could consider the clustering structure as an organic component that can evolve and change as a reaction to some triggers and decisions. For example, a company could decide to update the segmentation of its customers by re-running the segmentation's algorithms at regular interval using updated

\footnotetext{
*Corresponding author.

Email addresses: alex.seret@kuleuven.be (Alex Seret), smaldonado@uandes.cl (Sebastian Maldonado)
} 
characteristics of its customers or by adding new customers. Typical decisions are then made concerning how to make the structure evolve and whether or not to increase or decrease of the number of clusters. Analyzing these clusters' movements is studied by a domain called dynamic clustering in which dynamic patterns at the cluster-level are identified through time (see e.g. Peters, 2012). Such an analysis focuses on the impact of the evolution of the values of the customers' characteristics on the segmentation.

Contrasting to this, one could be interested in the impact of the evolution of the set of characteristics on the segmentation, which is the topic of this work. More especially, this paper discusses a particular case of such an evolution of the characteristics in a segmentation context. Considering a customer segmentation based on a set of variables, further referred as the original variables, this work aims at ranking variables from another set of new attributes, further called the candidate variables, based on their relevance for improving the original segmentation.

The business relevance of this work is further illustrated by a case study involving the marketing department of a main event organizer based in Europe. After performing a segmentation of their customers using the variables perceived at that moment as the relevant variables, the business involved integrated the obtained segments in their strategy and are still, at the moment of the writing of this paper, using these segments to guide their efforts. As mentioned above, different steps as dynamic clustering or segmentation maintenance through update are conceivable. This work reports experiments performed while trying to identify next relevant variables that could enrich the segmentation while considering the original segments as prior knowledge. This idea of prior knowledge used as input for a new analysis is the main justification of this research from a practical point of view. Facing the decision to re-segment from scratch or to enrich their current segmentation, the business involved opted for the later option. Inspired by other works as Seret et al. (2014b) and Wagstaff et al. (2001), a need for a methodology allowing to guide a new segmentation by selecting the appropriate variables using the results of an existing segmentation has been identified and discussed in this work. The original segmentation and the identification of the next relevant variables are described in detail in this work using real data and solving a real problem of the ticketing industry.

The scientific relevance of this work is threefold. First, in order to rank the candidate variables conditionally to the original segmentation, seven different candidate techniques from the literature are described and used as feature selection mechanisms. The results of the experiments are then evaluated using four different evaluation criteria for clustering. Second, a new feature selection algorithm designed for this problem is proposed and compared with the other techniques. Finally, the different steps are reported as a generic methodology that could be applied in other domains, opening new tracks for research.

The remainder of this paper is structured as follows. In Section 2, the theoretical background on unsupervised learning for customer segmentation is summarized, including a description of relevant evaluation metrics for clustering performance. Section 3 presents different feature selection techniques that are relevant in this work. The proposed feature selection framework for updating a current clustering model is presented in Section 4 . In Section 5, the proposed methodology is applied in a real case involving a marketing department from the event industry. Section 6 , concludes this paper and identifies new tracks for research. 


\section{Data clustering for customer segmentation}

Customer segmentation is an approach aiming at grouping similar customers in order to better understand and approach them. Widely discussed in the literature, segmentation exercises have been conducted in different contexts, allowing researchers to identify critical issues and best practices. In a recent work, Hiziroglu (2013) identifies five of these main critical methodological issues related to segmentation research and discusses different considerations related to it. The first category of issues mentioned in their work concerns the problem definition related issues, one of the major considerations of which is the selection of segmentation variables and models. In this paper, the aim is to identify new relevant variables using an existing segmentation of a customer base, which is strongly related to the segmentation variables and models selection consideration of Hiziroglu (2013) and further discussed in the remaining of the manuscript. In this specific section, the clustering approach used as segmentation technique in the application of Section 5 is briefly introduced while referring the reader to previous works discussing it into detail. Note that the focus of this paper is more on the identification of new relevant variables for segmentation than on the segmentation technique itself, which explains the limited space used to discuss it while referring the reader to interesting related works. In order to allow for an evaluation of the resulting clustering partitions, an introduction to four evaluation metrics widely discussed in the literature is added to this section.

\subsection{The two-step clustering approach}

The clustering approach used in this work is based on a two-step clustering strategy discussed and applied in works as Louis et al. (2013), Seret et al. (2012), Azcarraga et al. (2005) and Vesanto and Alhoniemi (2000). The first step of this strategy consists of reducing the number of data points by training a self-organizing map (SOM) with a high number (lower than the number of input data points while being significantly higher than the expected number of clusters) of neurons (see Kohonen, 1995, for more details). By doing this, prototypes of the original data points are obtained and preserve the topology of the data in the original space. Combining both quantization and visualization facilities offered by the SOM algorithm, it is then possible to represent and visualize the structure of the data while capturing the main patterns with a second clustering step. During this second step, the neurons previously trained are clustered using a classical partitioning algorithm as the $k$-means algorithm (Tan et al., 2006). Since the number of neurons is significantly lower than the original number of data points while preserving the dimensionality, more computationally expensive experiments can be conducted as discussed in Vesanto and Alhoniemi (2000) and illustrated in Section 5. As an output of this two clustering steps, a mapping between the original data and the neurons and between the neurons and the clusters of the second step allow to cluster the original data points and to obtain cluster centers represented in the original space. This strategy is summarized on Figure 1 and applied in Section 5. The reader is referred to Kohonen (1995) for an exhaustive discussion of the SOM algorithm and to the previously cited works for examples of applications.

\subsection{Clustering evaluation metrics}

When facing a partitioning of data points resulting from a crisp clustering algorithm as the one presented in the previous section, internal clustering validation measures have to be considered in order to evaluate the quality of the obtained output. Different measures are proposed in the literature, a good summary of which is discussed in 


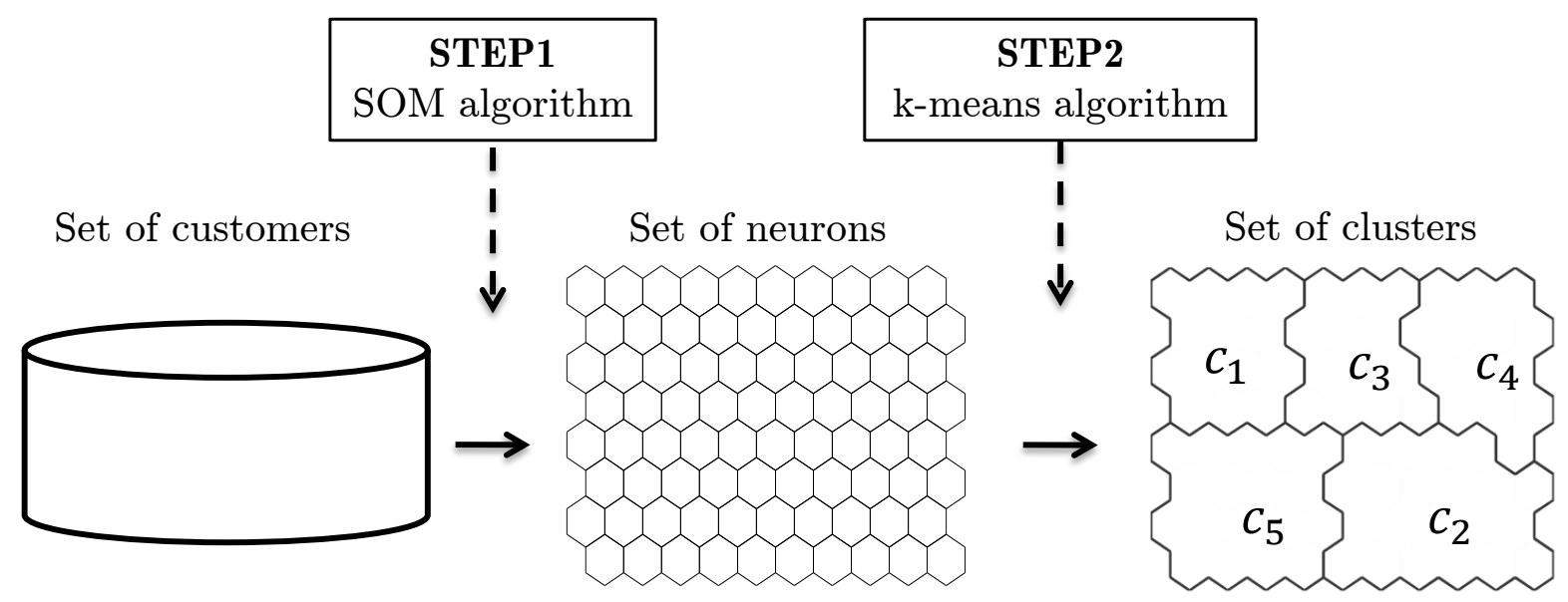

Figure 1: The 2-step clustering strategy.

Aggarwal and Reddy (2014). Although these evaluation metrics have specific characteristics, two general evaluation criteria can be identified. The first criterion is the compactness of a partitioning, which measures how closely related the objects in a cluster are. By reducing the variance within the different clusters of a partitioning, a higher compactness is obtained, resulting in a better clustering. The second general criterion is the separation, reflecting how well-separated or distinct a cluster is from other clusters. Creating partitions with a high intercluster dissimilarity, hence being well-separated, leads to clusters showing unique characteristics, which improves the general quality of the clustering. These two general criteria are key concepts leading the design of multiple clustering algorithms and evaluation metrics. In what follows, four metrics widely used and discussed in the literature are briefly introduced and positioned with regard to the two general criteria of compactness and separation. These evaluation metrics are further used in Section 5 in order to evaluate the different partitions resulting from the different feature selection approaches.

The first evaluation metric is the root-mean-square standard deviation (RMSSTD) of a partition $\mathcal{K}$ (a set of clusters $\mathcal{C}_{i}$, with $i=1, \ldots, N C$ ) calculated as:

$$
\operatorname{RMSSTD}(\mathcal{K})=\sqrt{\frac{\sum_{i} \sum_{x \in \mathcal{C}_{i}} d\left(x, c_{i}\right)^{2}}{p \sum_{i}\left(n_{i}-1\right)}},
$$

with $x$ being one of the $n$ objects of the set $\mathcal{N}$ of all samples. Each element of $\mathcal{N}$ belong to a given cluster $\mathcal{C}_{i}$ with center $c_{i}, n_{i}$ being the number of objects in cluster $\mathcal{C}_{i}, p$ being the number of variables in the model, and $d\left(x, c_{i}\right)$ being the Euclidean distance between an object $x$ and the centroid $c_{i}$. This metric evaluates the homogeneity of the formed clusters and is hence focusing on the compactness criterion.

The next metric is the R-squared (RS) of a partition $\mathcal{K}$ calculated as:

$$
R S(\mathcal{K})=\sqrt{\frac{\sum_{x \in \mathcal{N}} d(x, c)^{2}-\sum_{i} \sum_{x \in \mathcal{C}_{i}} d\left(x, c_{i}\right)^{2}}{\sum_{x \in \mathcal{N}} d(x, c)^{2}}} .
$$

By calculating the ratio of the sum of squares between clusters to the total sum of squares of the whole data set, an evaluation of the degree of difference between the clusters is obtained, hence evaluating the separation criterion. The next metric, the Calinski-Harabasz index $(\mathrm{CH})$, combines both the separation and compactness criteria by 
taking into account the between- and within-cluster sum of squares. The $\mathrm{CH}$ of a partition $\mathcal{K}$ is calculated as:

$$
C H(\mathcal{K})=\frac{\frac{\sum_{x \in \mathcal{N}} d(x, c)^{2}}{N C-1}}{\frac{\sum_{i} \sum_{x \in \mathcal{C}_{i}} d\left(x, c_{i}\right)^{2}}{n-N C}},
$$

with $c$ being the center of $\mathcal{N}$. The fourth metric considered in this paper is the Davies-Bouldin index (DB) of a partition $\mathcal{K}$ calculated as:

$$
D B(\mathcal{K})=\frac{1}{N C} \sum_{i} \max _{i^{\prime}, i^{\prime} \neq i}\left(\frac{\frac{1}{n_{i}} \sum_{x \in \mathcal{C}_{i}} d\left(x, c_{i}\right)+\frac{1}{n_{i^{\prime}}} \sum_{x \in \mathcal{C}_{i^{\prime}}} d\left(x, c_{i^{\prime}}\right)}{d\left(c_{i}, c_{i^{\prime}}\right)}\right) .
$$

Each cluster $\mathcal{C}_{i}$ is assigned the highest similarity between this cluster and all other clusters. The DB index is then the average of these different similarities, a low value meaning that clusters are very distinct, hence focusing on the separation criterion.

Although these four metrics only represent a subset of the available metrics (see Aggarwal and Reddy, 2014, for additional references), combining them to evaluate a partitioning allows us to obtain a clear idea of the output quality. Note that since the scales of the different metrics are different and difficult to interpret as such, a relative ranking of different clustering results based on the different metrics is preferable. For example, consider two partitions $\mathcal{K}^{1}$ and $\mathcal{K}^{2}$ obtained after clustering a data set $\mathcal{N}$. If $\operatorname{RMSSTD}\left(\mathcal{K}^{1}\right)$ is greater than $\operatorname{RMSSTD}\left(\mathcal{K}^{2}\right)$, $R S\left(\mathcal{K}^{1}\right)$ is smaller than $R S\left(\mathcal{K}^{2}\right), C H\left(\mathcal{K}^{1}\right)$ is smaller than $C H\left(\mathcal{K}^{2}\right)$ and $D B\left(\mathcal{K}^{1}\right)$ is greater than $D B\left(\mathcal{K}^{2}\right)$, we may say that $\mathcal{K}^{2}$ is a better partitioning than $\mathcal{K}^{1}$. In some cases, different metrics may lead to different conclusions, which motivates the use of more than one metric to assess the relative quality of a clustering output.

\section{Feature ranking techniques for unsupervised and supervised learning}

In this section we briefly describe the feature ranking approaches used in this work to sort the candidate variables according to their relevance, given a current clustering partition obtained using the original variables. Feature ranking for unsupervised learning aims at ranking these candidate variables based on feature extraction techniques, taking into account the redundancy between the candidate variables. Principal Component Analysis and Generalized Hebbian Algorithm (Lee and Verleysen, 2007) are used to extract one component from the correlation matrix created with all candidate variables, while these variables are ranked according to their correlation with this component. Supervised feature ranking is performed by assessing each candidate variable's correlation with the labels (in our case, the current partition). This can be approached either in a bivariate fashion (using e.g. methods as Fisher Score, Chi Square, and Information Gain), or using multivariate methods (using e.g. methods as RELIEFF and Random Forest). A brief description of these different techniques is reported in what follows in order to provide the reader with a basic understanding of the main concepts. These techniques are further used in Section 5 as benchmark for the proposed approach.

\subsection{Fisher Score}

A commonly used filter method is the Fisher Criterion Score $(F)$ (Duda et al., 2001), which assesses each feature's importance by computing the correlation between each candidate variable $j \in \mathcal{D}$, where $\mathcal{D}$ is the set of the candidate variables, and the output labels given by the current clustering partition: 


$$
F(j)=\frac{\sum_{i=1}^{N C} n_{i}\left(\mu_{i, j}-\mu_{j}\right)^{2}}{\sum_{i=1}^{N C} n_{i} \sigma_{i, j}^{2}},
$$

where $\mu_{j}$ represents the mean of variable $j, \mu_{i, j}$ and $\sigma_{i, j}$ are the mean and standard deviation for variable $j$ on cluster $i$, respectively, and $n_{i}$ is the number of elements that belong to cluster $i=1, \ldots, N C$.

\subsection{Chi Square test}

The Pearson's $\chi^{2}$ independence test determines whether the distribution of paired observations on two categorical variables, expressed in a contingency table, are similar to each other (Pearson, 1900). The test is the sum of the squared difference between observed and the expected (or theoretical) frequency that holds under the hypothesis of independence, divided by the expected frequency in all possible levels (numerical candidate variables are previously binned into $R$ levels):

$$
\chi^{2}(j)=\sum_{r=1}^{R} \sum_{i=1}^{N C} \frac{\left(n_{i, r}-\mu_{i, r}\right)^{2}}{\mu_{i, r}}
$$

where $\mu_{i, r}=\frac{n_{* i} n_{r *}}{n}$ is the expected fraction of samples for the $r$-th feature category of variable $j$ and cluster $i$, and $n_{i, r}$ is the number of examples in the $r$-th level of variable $j$ that belongs to cluster $i$.

\subsection{Information Gain}

The information gain corresponds to the change of information entropy by including the variable, with respect to the baseline entropy of the dataset (Yang and Pedersen, 1997). The information gain of a variable $j$ that has been binned into $R$ levels is defined as:

$$
I G(j)=-\sum_{i=1}^{N C} P(i) \log P(i)+\sum_{r=1}^{R} P(r) \sum_{i=1}^{N C} P(i \mid r) \log P(i \mid r),
$$

where $P(\cdot)$ is the ratio of the particular category in the dataset: $P(i)=\frac{n_{i}}{n}, P(r)=\frac{n_{r}}{n}$, with $n_{r}$ being the number

of examples that belong to level $r$, and $P(i \mid r)=\frac{n_{i, r}}{n_{r}}$ being the fraction of samples of level $r$ that belongs to cluster $i$.

\subsection{Random Forests}

Feature selection can be performed with tree-based ensembles, such as Random Forests, which inherit all nice properties of single tree while providing a more reliable ranking since the relevance measure is averaged over the $N$ trees in the ensemble (Breiman, 2001). Considering a tree that uses information gain, the variable importance can then be defined as the sum over the tree nodes $t$ (Guyon et al., 2006):

$$
M(j)=\sum_{t \neq T} I G(j, t) .
$$

The variable importance at the ensemble-level is then the average $M(j)$ over the $N$ trees in the ensemble:

$$
R F(j)=\frac{1}{N} \sum_{k=1}^{N} M_{k}(j)
$$




\subsection{RELIEFF}

RELIEFF is an iterative algorithm that estimates the quality of the variables according to how well their values distinguish between objects that are near to each other (Kononenko, 1994). For a randomly selected example $\mathbf{x}$, the method searches for its nearest neighbors: one from the same class (near-hit) and one for every opposite class (near-miss). The algorithm iteratively updates a weight vector $W$ for all attributes by subtracting the distance between the randomly selected example $\mathbf{x}$ and its near-hit, and adding the weighted distances to the near-miss of every opposite class, as follows:

$$
W:=W-(\mathbf{x}-N H)^{2}+\sum_{i \neq \operatorname{class}(\mathbf{x})} \frac{P(i)\left(\mathbf{x}-N M_{i}\right)^{2}}{m},
$$

where $N H$ and $N M_{i}$ respectively denotes the near-hit and near-miss to every opposite cluster $i$. The rationale behind this approach is that a relevant attribute should differentiate between examples from different classes (clusters in our case) and should have similar values for samples from the same class (Kononenko, 1994). These weights are used as a relevance vector for feature ranking after a predefined number of iterations.

\subsection{Feature Ranking via PCA and GHA}

Extraction can be used as a filter technique for unsupervised feature selection. Different methods have been proposed in the literature to perform such extraction of components: Principal Component Analysis (PCA), Kernel PCA, Singular Value Decomposition (SVD), non-linear PCA, and the Generalized Hebbian Algorithm (GHA); see Lee and Verleysen (2007) for an overview of the respective approaches. These methods can be adapted to rank the original attributes in terms of their influence in the components. In particular, we use the weights associated with each attribute that constructs the first component to rank the variables in terms of relevance, where the higher the weight of an attribute in magnitude the more relevant it is considered.

In this paper the Principal Component Analysis (PCA) and the Generalized Hebbian Algorithm (GHA) are used for feature ranking. PCA performs feature extraction in such a way that the first principal component has the largest possible variance, resulting in the eigenvector associated to the largest eigenvalue of the covariance matrix. Each succeeding component has the highest variance possible under the constraint that it is orthogonal to the preceding components. A detailed discussion about PCA can be found in Lee and Verleysen (2007).

GHA has been presented in Sanger (1989) and generalizes the learning rule proposed in Hebb (1949). Inspired by insights from synaptic plasticity in neuroscience, this rule basically says that the weight between two neurons increases if both neurons are active at the same time. Applying this idea to the extraction of principal components can be formalized as follows. First, the single output neuron of a one-layer feedforward neural network $y$ that represents the first principal component is computed as follows:

$$
y=\sum_{j \in \mathcal{D}} w_{j} x_{j}
$$

where $x_{j}$ represents input neuron (attribute) $j$ and $w_{j}$ its respective weight. The following rule is used to update the weights: 


$$
w_{j}:=w_{j}+\eta y\left(x_{j}-y w_{j}\right),
$$

where $\eta$ is a positive learning rate. GHA offers certain advantages over PCA since it determines the components in an iterative way rather than calculating the covariance matrix as is the case in PCA.

\section{The proposed feature ranking methodology}

The proposed methodology is a filter approach that ranks a set of candidate variables $\mathcal{D}$ using the information of an existing set of clusters $\mathcal{K}$. To do so, two of the main concepts related to the intrinsic quality of a partitioning are considered: namely the compactness and the separation of the resulting partition. The idea here is to sort the variables according to their capacity to form compact clusters that are well separated from other clusters. In order to assess the individual variables instead of the whole partitioning (as traditionally done), we introduce the concept of compactness at an attribute-level, Comp $p_{j}$, and the concept of separation at an attribute-level, $\operatorname{Sep}_{j}$; $j$ being a variable of $\mathcal{D}$. In order to calculate these two measures, different intermediary steps are necessary, as explained in what follows.

Recalling the notation presented for the method Fisher Score in the previous section, we define $\mu_{i, j}$ as the mean for candidate variable $j$ on cluster $i$, where $\mu_{i, j}=\frac{\sum_{x \in \mathcal{C}_{i}} x_{j}}{n_{i}}$. Additionally, we refer to $\mu_{i, j}^{C}$ as the mean value for variable $j$ of all objects that do not belong to cluster i, i.e. $\mu_{i, j}^{C}=\frac{\sum_{x \in \mathcal{N} \backslash \mathcal{C}_{i}} x_{j}}{\left|\mathcal{N} \backslash \mathcal{C}_{i}\right|}$, where $\left|\mathcal{N} \backslash \mathcal{C}_{i}\right|$ is the cardinality of the set of all elements but excluding those that belongs to cluster $i$.

Based on previous definitions, we compute the compactness of a variable $j$ as:

$$
\mathrm{Comp}_{j}=\frac{1}{N C} \sum_{i \in \mathcal{K}}\left(\frac{1}{n_{i}} \sum_{x \in \mathcal{C}_{i}} a b s\left(x_{j}-\mu_{i, j}\right)\right)
$$

and the separation of a variable $j$ as:

$$
\operatorname{Sep}_{j}=\frac{1}{N C} \sum_{i \in \mathcal{K}} a b s\left(\mu_{i, j}-\mu_{i, j}^{C}\right),
$$

where $a b s(f)$ is the absolute value of $f$. The variables in $\mathcal{D}$ are sorted using this two criterion, creating two different rankings. A first ranking, $L C$, contains the variables in $\mathcal{D}$ sorted according to their compactness, starting with the variable with the lowest value. A second list, $L S$, contains the variables in $\mathcal{D}$ sorted according to their separation, starting with the variable with the highest value. For both lists, ties are solved randomly. As a final step, considering a function $p(L, j)$ returning the position of a variable $j$ in a list $L \in\{L C, L S\}$, the final ranking of the variables of $\mathcal{D}, C S(\mathcal{D})$, is a list of the variables of $\mathcal{D}$ sorted according to both their separation and compactness, starting with the variables with the lowest values of $\operatorname{pos}(L C, j)+\operatorname{pos}(L S, j)$, solving ties randomly.

The result of this approach is a sorted list of the variables of $\mathcal{D}$ conditionally to the previously obtained clustering $\mathcal{K}$. The first variables of this ranking are variables with a high score for the compactness and a low score for the separation. This approach is used as a feature selection mechanism in Section 5 and compared to the alternative feature ranking approaches introduced in the previous section. A summary of the main steps of this approach is proposed in Algorithm 1. 


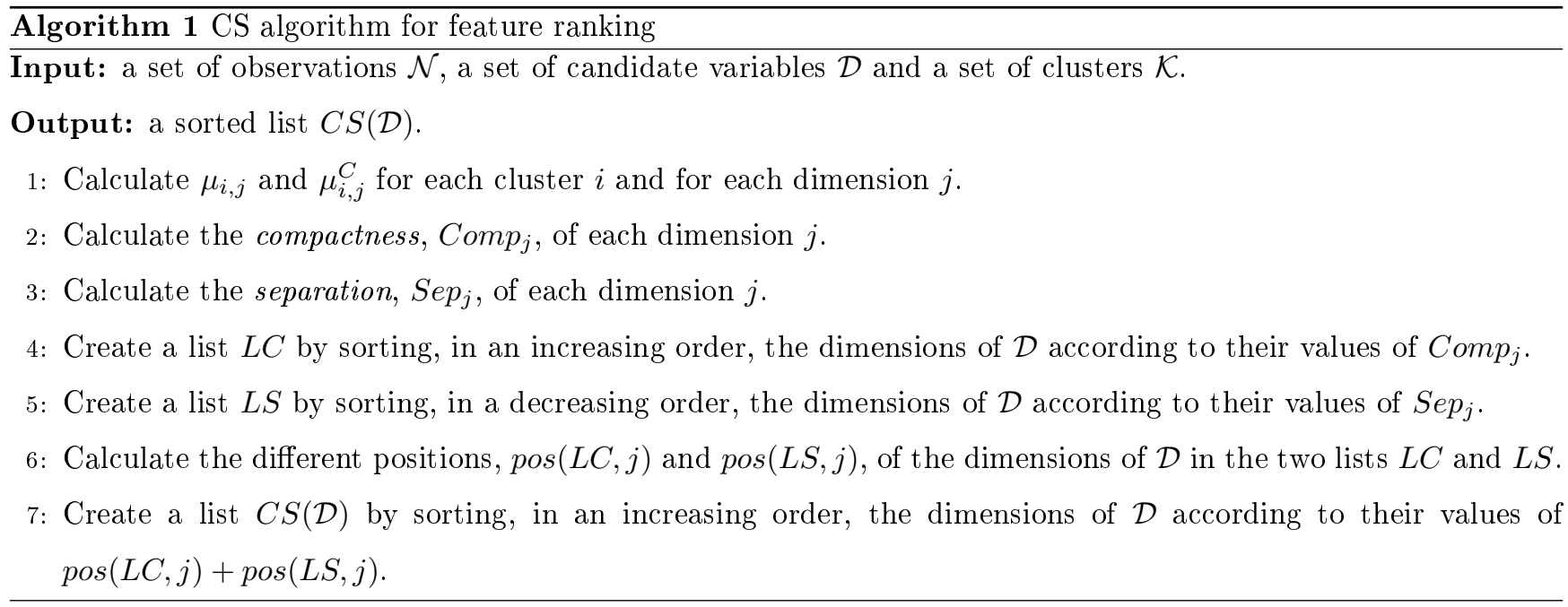

\section{Application}

In this section, an application in the concert industry is reported and discussed in detail in order to guide the reader through the different steps taken by the business and researchers involved in this project. The business consists of members from the marketing department of a main event organizer based in the Netherlands that collaborated with the authors in order to better understand their customer base and obtain actionable segments that would be integrated in their marketing strategy. After obtaining a segmentation of their customers based on meaningful variables, new variables are created and evaluated in order to be incorporated into the existing segmentation. This section consists of two main sub-sections. In Section 5.1, the different steps leading to the original segmentation of the customer base are presented in detail. Section 5.2 describes how new relevant variables can be identified in order to complete and improve the segmentation obtained in Section 5.1. Note that the different concepts and techniques used in this section are introduced in Sections 2, 3 and 4.

\subsection{Original segmentation of the customer base}

The customers of the company involved in this application, called EventOrga in the remainder of this paper, are interacting with the company through different channels. Thanks to the collaboration of different technology providers, these interactions enable the capture of data that further can be used to understand the customers. In this section, the different steps leading to the creation of segments of customers are described. This segments are currently used by EventOrga in order to guide their marketing efforts on a daily basis.

Two main sources of data are used in order to create the variables based on which customer segments are formed. The first data source consists of six years of ticketing data gathering the logs of the transactions made by the different customers. Demographic data about the customers and transactional data at the level of tickets are captured and aggregated at the customer level in order to fuel this database. Note that a customer, in this context, is the person buying the tickets and is not especially the person attending the concert. However, in this paper, it is said that a customer attended a concert if he bought a ticket for it and the concert took place in the past. A second data source consists of a product database in which the characteristics of the different products (events in this case) are stored. Because the transactional logs are linked to specific events, both databases can be 
combined in order to create rich and meaningful variables for the business. The way these variables are calculated is shortly explained in what follows. First the well known RFM variables (Recency, Frequency and Monetary) are calculated (Colombo and Jiang, 1999). The variable Recency is obtained by calculating the delta, in days, between the last concert attended by a customer and the moment of the calculation of this variable. The output of this variable is a number of days. The variable Frequency corresponds to the weighted number of concerts attended by a customer. The weights are business input assigned to the different seasons ${ }^{1}$ and reflect the fact that a customer with a high frequency of visits during the last season should have a higher value for Frequency than a customer with the same frequency of visits but during an older season. The output of this variable is a positive number reflecting the weighted frequency of visits of a customer. The variable Monetary is calculated as the average money spent by season. The output is an amount in euro. Three variables (OnlyFuture, FutureEvent and Trend) linked to the RFM variables are then calculated. The variable OnlyFuture is equal to 1 if a customer purchased tickets only for future events (the customer is in the database but his associated events are in the future) and is equal to 0 otherwise. The variable FutureEvent is equal to 1 if a customer has tickets for at least one future event and is equal to 0 otherwise. The variable Trend is obtained by dividing the number of active seasons ${ }^{2}$ of a customer by the length of his relationship, expressed in seasons, with EventOrga. The next variable, DayDelta, captures the average number of days separating the purchase of a ticket for an event and the event itself. The output is a number of days. The variable Distance captures the geodesic distance in kilometers separating the customer (based on his address) and the event infrastructure. The variable FirstLog expresses, in years, the duration of the relationship between the customer and EventOrga. The variable NbTickets is calculated as the average number of tickets per event, the output being a number of tickets. Finally, two variables representing ratios are calculated. The variable SubscriptionRatio is calculated as the number of tickets purchased using a subscription divided by the total number of tickets purchased by a customer while the variable ExternalRatio captures the ratio of external concerts ${ }^{3}$ to the total number of concerts attended by a customer. The different variables, their ranges and units are reported in Table 5.1.

After defining the different variables, a period of time is selected and data is gathered in order to calculate the values of the different variables for the customers active during this period. For this segmentation, a period of six years has been selected by the business based on operational considerations related to the quality of the data. The different data sources necessary to create the variables of Table 5.1 have been collected and cleaned using statistical tools and the business expertise. Note that the removal of outliers in the database has been a very important preprocessing step for this analysis. Although statistical tools can support this process, collecting experts' insight is a crucial step in the preprocessing phase. For example, after analyzing the available data, it has been decided to remove some of the customers because they were representatives from companies and hence showing unusually high RFM values. Deciding whether or not to keep these customers is a subjective but important step in the quest of value-adding segments and depends on business expectations and goals. After preprocessing, a total of 82082 customers are selected. These customers are customers active during the six last years. The variables of

\footnotetext{
${ }^{1}$ A season is a period of 12 months starting on the $1^{\text {st }}$ of August.

${ }^{2} \mathrm{An}$ active season is a season during which a customer attended at least one event.

${ }^{3}$ An external concert is a concert programmed by an external organization but taking place in the infrastructure of EventOrga.
} 


\begin{tabular}{lll}
\hline Variable & Range & Unit \\
\hline Recency & {$[0, \infty)$} & days \\
Frequency & $(0, \infty)$ & weighted frequency \\
Monetary & {$[0, \infty)$} & euro \\
OnlyFuture & {$[0,1]$} & dummy \\
FutureEvent & {$[0,1]$} & dummy \\
Trend & $(0,1]$ & ratio \\
DayDelta & {$[0, \infty)$} & days \\
Distance & {$[0, \infty)$} & km \\
FirstLog & {$[0, \infty)$} & years \\
NbTickets & $(0, \infty)$ & number of tickets \\
SubscriptionRatio & {$[0,1]$} & ratio \\
ExternalRatio & {$[0,1]$} & ratio \\
\hline
\end{tabular}

Table 1: Summary of the different variables used as input for the original segmentation, together with their ranges and units.

Table 5.1 are calculated for each of them by only using data of this period, except for the variable FirstLog for which older data sources are also considered in order to better reflect the reality. As the objective of this section is to create segments within the customer base (i.e. within the 82082 customers previously obtained), groups have to be identified such that customers within a group are relatively similar to other customers in that group while being relatively dissimilar to customers in other groups. To do so, two clustering steps are performed as described in what follows.

The first clustering step consists of the application of the SOM algorithm in order to summarize the 82082 customers characterized by the variables of Table 5.1 after standardization. The goal is to summarize the input data by using a relatively high number of neurons, allowing data summarization, visualization and noise removal. By applying this first step, a first exploration of the data is possible as illustrated in Figure 2 where the component planes of a $20 \times 25 \mathrm{SOM}$ are depicted. The choice of the number of neurons and the shape of the maps are following best practices. It is indeed recommended to use a number of neurons significantly higher than the expected number of clusters while preferring a rectangular shape in order to facilitate the projection (Kohonen, 1995). This explains why a $20 \times 25 \mathrm{SOM}$, instead of e.g. a $10 \times 10 \mathrm{SOM}$, is chosen. Each component plane of Figure 2 represents the values for the different neurons of the respective variable using a color code ranging from dark blue (low values) to dark red (high values). For example, by referring to Figure 2(a), the component plane of variable Recency, it can be said that neurons at the top right corner of the map are representing neurons, hence prototypes of customers, with relatively high values for the variable Recency. By combining multiple component planes, different conclusions can be drawn. For example, by referring to the bottom right corners of Figures 2(b) and 2(c), it can be said that some neurons, hence a group of customers, have a specific characteristic being relatively high values for the variables Frequency and Monetary. By identifying such visual patterns, the analyst is able to get first insights at 


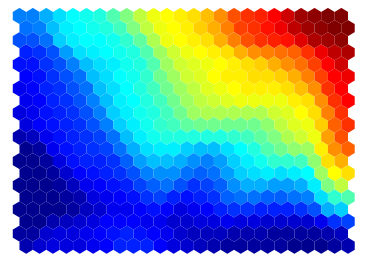

(a) Recency

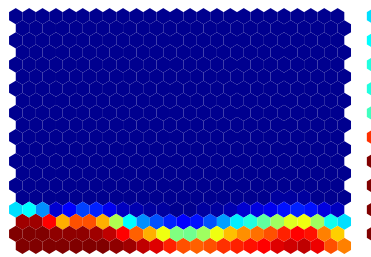

(e) FutureEvent

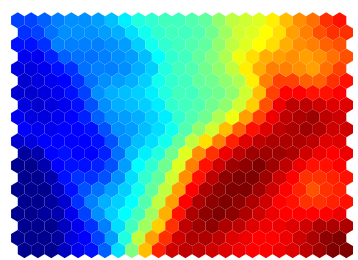

(i) FirstLog

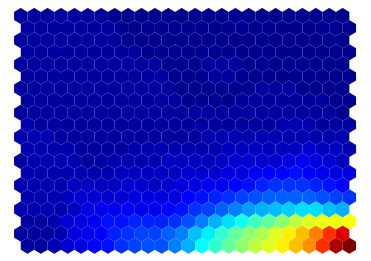

(b) Frequency

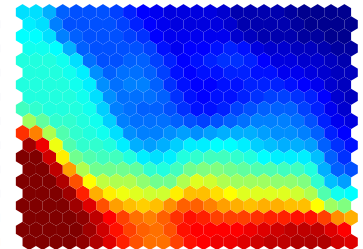

(f) Trend

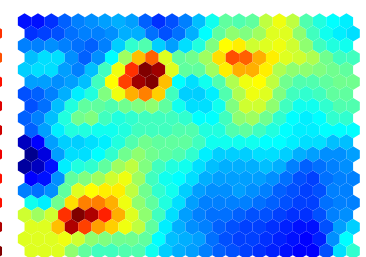

(j) NbTickets

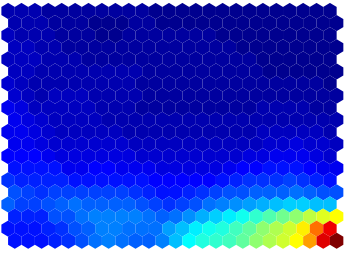

(c) Monetary

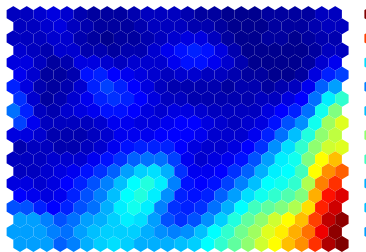

(g) DayDelta

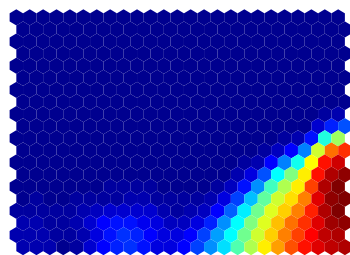

(k) SubscriptionRatio

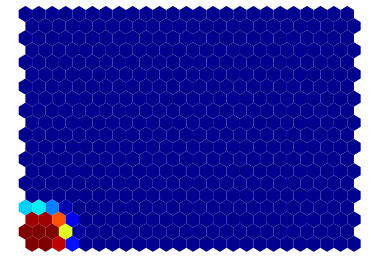

(d) OnlyFuture

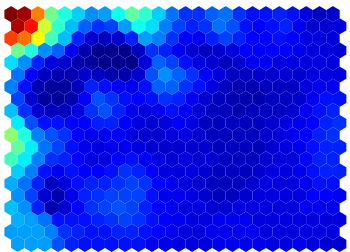

(h) Distance

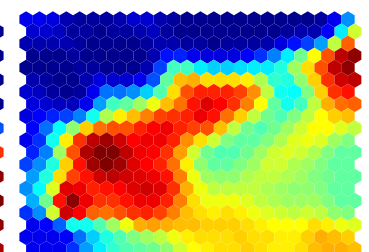

(1) ExternalRatio

Figure 2: The 12 component planes obtained after applying the SOM algorithm on the original variables.

an early stage of the segmentation, allowing him to perform, if necessary, some corrective actions. Although such visual analysis can be useful to make a first exploration of the data by e.g. visually identifying outliers or potential clusters, a second clustering step is necessary to obtain a manageable number of clusters that can be described and reported statistically. Note that this first clustering step allows the analyst to continue the analysis with 500 neurons $\left(20^{*} 25\right)$ instead of the 82082 customers, which eases both manipulation and computational efforts during the second clustering step as mentioned in the theoretical section. To summarize, the customers are first represented by a high number of neurons that are further clustered.

As a second clustering step, the neurons trained with the SOM algorithm are used as input for the $k$-means algorithm (note that the weights of the SOM output are already based on standardized variables such that no standardization or normalization is performed for this second clustering step). The maximum number of clusters, $k^{M A X}$, is set to 20 based on business constraints and first insights obtained during the visual exploration using the component planes. Using the Davies-Bouldin index to select the best partition, the 20 clusters $\left(c_{1}\right.$ to $\left.c_{20}\right)$ of Figure 3 are selected. The centroids, or centers, of these 20 clusters are reported in Figure 4 (after returning to the original scales). The color code used reflects the relative values within each variable of the different clusters; the cluster having the highest value for a given variable being colored in dark green while the lowest value is represented in dark red. Note that cluster $c_{12}$ has no value for the variable Recency, which makes sense since the value of the variable OnlyFuture is equal to one, meaning that this cluster captures customers whose first events are in the future, such that the Recency value can not be calculated. The number of customers and the percentage of the customer base it represents are also reported, thanks to a mapping between customers and neurons and between 


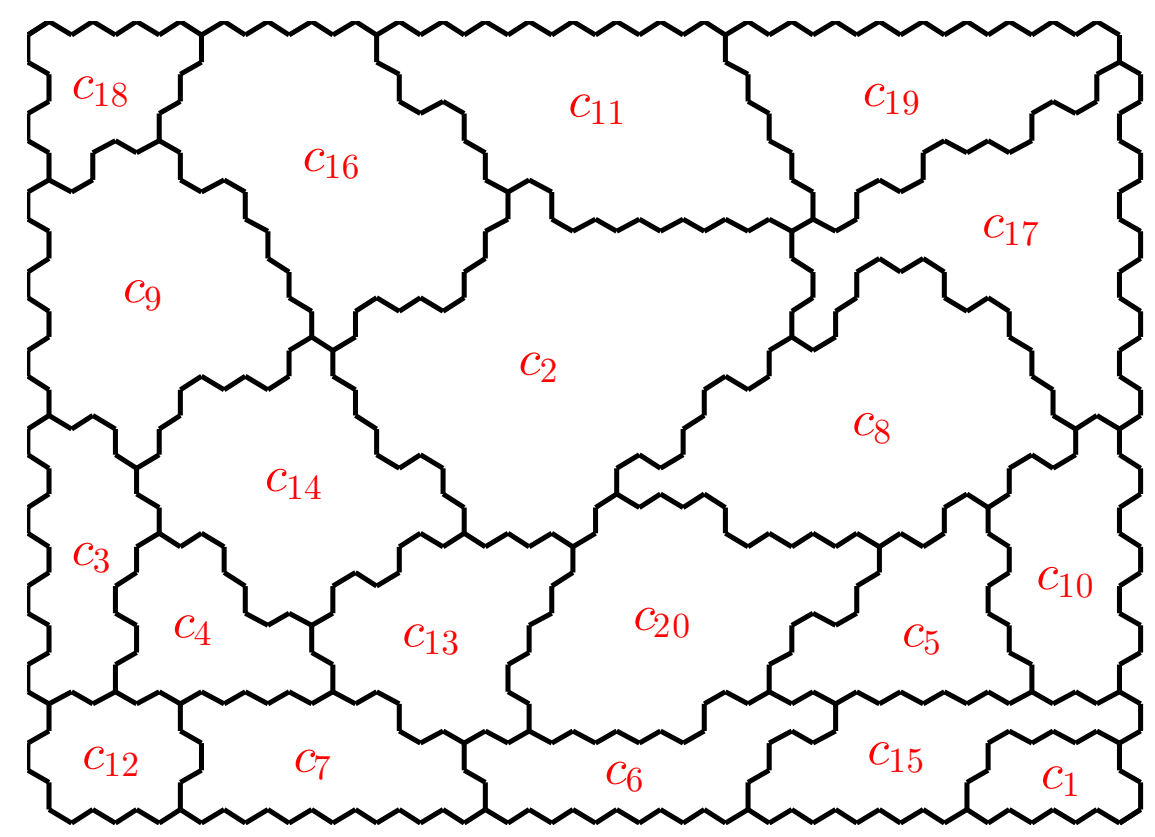

Figure 3: The 20 clusters obtained after applying the $k$-means algorithm on the output of the SOM algorithm.

neurons and clusters. It is indeed important to note that neurons are prototypes of customers and clusters are groups of neurons. It is thus possible to link the customers to their BMUs (closest neurons) and these BMUs to their closest clusters, hence linking the clusters back to the customers. The centroids of Figure 4 are representing the average values per cluster of the different variables of Table 5.1. Although the second clustering step is based on the neurons, the values reported are the averages at the customer-level in order to better reflect the reality. The different outputs of this section are used in the daily practice of EventOrga, especially Figure 4 which provides them with an advanced and detailed segmentation output based on meaningful variables.

Different next steps can then be considered by EventOrga in order to build on this first exercise and better understand their customers. On the one hand, other data mining techniques as dynamic clustering approaches (see e.g. Seret et al., 2014a; Peters, 2012) or recommender systems can be applied and combined with the output of this segmentation, hence using it as a new knowledge source for future analyses. On the other hand, the current segmentation could also be enriched by considering new data sources while building on the original segmentation as illustrated in the next section.

\subsection{Identification of new variables}

In this section, the techniques proposed in the theoretical sections are applied in order to identify variables to add to the existing segmentation. As it is often the case in practice, a segmentation task is based on a set of variables considered as relevant at the beginning of the analysis. Although these variables may remain relevant in the future, new variables could be later considered to enrich the segmentation of the customers. This situation can be approached in two different ways. A first approach could consist of a new segmentation based on both sets of variables. However, while this approach is typically considered in practice, the method proposed in this paper aims at ranking the new set of variables based on how it could enrich the original set of variables and improve the segmentation results. The main advantage of this approach is that it allows the practitioner to continue with 


\begin{tabular}{|c|c|c|c|c|c|c|c|c|c|c|c|c|c|c|}
\hline & 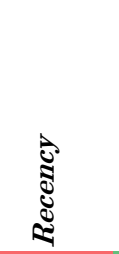 & 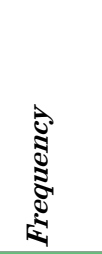 & 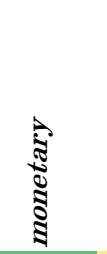 & 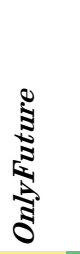 & 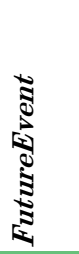 & 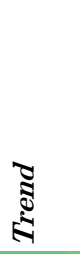 & 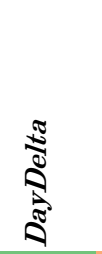 & 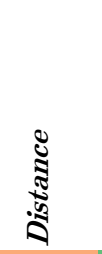 & 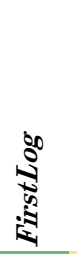 & 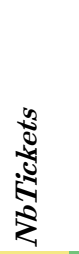 & 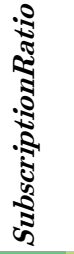 & 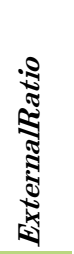 & 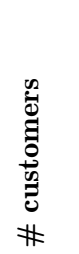 & $\%$ \\
\hline c1 & 61.55 & 205.57 & 549.95 & 0.00 & 0.98 & 0.99 & 259.67 & 15.89 & 6.73 & 2.45 & 0.79 & 0.65 & 2425 & 2.95 \\
\hline c2 & 887.39 & 5.34 & 26.52 & 0.00 & 0.00 & 0.32 & 35.14 & 23.44 & 2.83 & 2.42 & 0.00 & 0.93 & 7058 & 8.60 \\
\hline c3 & 68.18 & 7.52 & 81.58 & 0.00 & 0.00 & 0.99 & 41.62 & 41.47 & 0.37 & 2.02 & 0.01 & 0.03 & 2494 & 3.04 \\
\hline c4 & 105.04 & 11.91 & 99.69 & 0.00 & 0.00 & 0.96 & 32.45 & 19.10 & 0.87 & 3.21 & 0.02 & 0.88 & 1199 & 1.46 \\
\hline c5 & 430.53 & 47.68 & 136.22 & 0.00 & 0.00 & 0.67 & 149.35 & 15.92 & 5.88 & 2.15 & 0.58 & 0.49 & 1827 & 2.23 \\
\hline c6 & 213.29 & 68.07 & 193.21 & 0.00 & 0.85 & 0.87 & 124.67 & 17.29 & 6.52 & 2.28 & 0.12 & 0.53 & 977 & 1.19 \\
\hline c7 & 303.90 & 27.47 & 117.78 & 0.00 & 1.00 & 0.88 & 121.06 & 21.35 & 2.02 & 2.52 & 0.14 & 0.33 & 1610 & 1.96 \\
\hline c8 & 859.72 & 8.15 & 32.33 & 0.00 & 0.00 & 0.39 & 48.46 & 11.87 & 6.33 & 2.28 & 0.01 & 0.28 & 5041 & 6.14 \\
\hline c9 & 316.91 & 6.25 & 40.02 & 0.00 & 0.00 & 0.51 & 43.02 & 17.59 & 1.12 & 2.23 & 0.00 & 0.01 & 6308 & 7.68 \\
\hline c10 & 1020.35 & 22.60 & 77.95 & 0.00 & 0.00 & 0.38 & 283.67 & 21.93 & 5.65 & 2.29 & 0.88 & 0.39 & 4306 & 5.25 \\
\hline c11 & 1143.38 & 3.61 & 19.59 & 0.00 & 0.00 & 0.26 & 38.35 & 30.47 & 3.44 & 2.63 & 0.00 & 0.01 & 9045 & 11.02 \\
\hline c12 & & 7.11 & 83.80 & 1.00 & 1.00 & 1.00 & 104.78 & 36.65 & 0.34 & 2.88 & 0.03 & 0.10 & 1881 & 2.29 \\
\hline c13 & 423.99 & 12.01 & 96.58 & 0.00 & 0.00 & 0.54 & 200.21 & 34.01 & 2.72 & 2.64 & 0.01 & 0.92 & 1591 & 1.94 \\
\hline c14 & 333.62 & 7.27 & 45.72 & 0.00 & 0.00 & 0.52 & 30.72 & 22.14 & 1.23 & 2.34 & 0.01 & 0.94 & 3936 & 4.80 \\
\hline c15 & 125.43 & 103.30 & 257.54 & 0.00 & 0.84 & 0.91 & 237.46 & 17.67 & 5.74 & 2.13 & 0.70 & 0.49 & 1840 & 2.24 \\
\hline c16 & 691.74 & 5.02 & 31.22 & 0.00 & 0.00 & 0.35 & 42.65 & 15.15 & 2.21 & 3.39 & 0.00 & 0.01 & 7537 & 9.18 \\
\hline c17 & 1512.36 & 2.77 & 16.16 & 0.00 & 0.00 & 0.22 & 69.81 & 20.27 & 5.31 & 2.54 & 0.00 & 0.84 & 5860 & 7.14 \\
\hline c18 & 599.03 & 5.79 & 39.79 & 0.00 & 0.00 & 0.42 & 51.17 & 119.67 & 2.04 & 2.13 & 0.01 & 0.16 & 4007 & 4.88 \\
\hline c19 & 1556.83 & 1.90 & 11.98 & 0.00 & 0.00 & 0.19 & 27.46 & 17.08 & 4.84 & 2.39 & 0.00 & 0.00 & 9043 & 11.02 \\
\hline c20 & 360.46 & 23.21 & 78.32 & 0.00 & 0.00 & 0.68 & 50.10 & 14.25 & 6.26 & 2.26 & 0.02 & 0.43 & 4097 & 4.99 \\
\hline
\end{tabular}

Figure 4: The centroids of the 20 clusters.

the original variables while considering the original segmentation as a prior knowledge guiding the selection of the new variables. By doing this, even if new segments of customers are obtained, these segments are aligned with the original segmentation since they are constructed based on the previously generated knowledge. Aligning the second segmentation with the first one while bringing new insights is believed to help the business to continue to follow the initiated strategy. Note that the goal of this section is not to provide the practitioner with a unique best segmentation but to provide a way to enrich a segmentation in a meaningful way. If new insights are generated by making a new segmentation, it is still important to keep the original segmentation in mind and consider the new segmentation as a complement and not as replacement. In order to illustrate the relevance of this problem, the case of the marketing team of EventOrga is considered. After a successful data mining exercise, the team obtained the 20 segments of Section 5.1 based on the 12 variables of Table 5.1 and decided to integrate it into its daily strategy and practices. As more and more data sources are getting available, new variables relevant for the business are identified and calculated after some months, which raises the question of how to integrate them considering the current segmentation. In what follows, different techniques discussed in this paper are applied in order to propose a solution to this problem by ranking a set of candidate variables conditionally to a segmentation based on an original set of variables. Since, to the best of the knowledge of the authors, this problem has never been approached or discussed in the literature, state-of-art existing techniques (introduced in Section 3) traditionally used in other contexts are assessed with regard to their ability to solve this problem. Additionally, a technique (proposed in Section 4) designed for this specific problem is applied and compared to the other alternatives. The best strategy 


\begin{tabular}{ll|ll}
\hline Variable & Range & Variable & Range \\
\hline FamilyConcertCount & {$[0, \infty)$} & MondayCount & {$[0, \infty)$} \\
JazzCount & {$[0, \infty)$} & TuesdayCount & {$[0, \infty)$} \\
JazzWorldMusicCount & {$[0, \infty)$} & WednesdayCount & {$[0, \infty)$} \\
ClassicalCount & {$[0, \infty)$} & ThursdayCount & {$[0, \infty)$} \\
PopularCount & {$[0, \infty)$} & FridayCount & {$[0, \infty)$} \\
RedSofaCount & {$[0, \infty)$} & SaturdayCount & {$[0, \infty)$} \\
WorldMusicCount & {$[0, \infty)$} & SundayCount & {$[0, \infty)$} \\
FamilyConcertRatio & {$[0,1]$} & MondayRatio & {$[0,1]$} \\
JazzRatio & {$[0,1]$} & TuesdayRatio & {$[0,1]$} \\
JazzWorldMusicRatio & {$[0,1]$} & WednesdayRatio & {$[0,1]$} \\
ClassicalRatio & {$[0,1]$} & ThursdayRatio & {$[0,1]$} \\
PopularRatio & {$[0,1]$} & FridayRatio & {$[0,1]$} \\
RedSofaRatio & {$[0,1]$} & SaturdayRatio & {$[0,1]$} \\
WorldMusicRatio & {$[0,1]$} & SundayRatio & {$[0,1]$} \\
\hline
\end{tabular}

Table 2: Summary of the different candidate variables, together with their ranges.

to adopt for this application is then selected and the candidate variables are ranked, allowing, inter alia, a new segmentation of the customers. These different steps are reported in detail in what follows, starting with a brief description of the candidate variables.

By making use of data from the product database, the preferences of the customers concerning the day and the genre of the events can be captured and new variables, the candidate variables, can be created. 14 variables capturing the preferences concerning the day of the events are first created. Firstly, the day of the concert is exploited and 7 variables, MondayCount, TuesdayCount, WednesdayCount, ThursdayCount, FridayCount, SaturdayCount and SundayCount, are calculated at the customer level and represent the number of concerts, attended by the customer, that took place on the respective days. Based on these 7 variables, 7 other variables, MondayRatio, TuesdayRatio, WednesdayRatio, ThursdayRatio, FridayRatio, SaturdayRatio and SundayRatio, are obtained by dividing each of them by the total number of concerts attended by the customer, hence obtaining ratios. Secondly, the genre of the concert is exploited and 7 main genres are identified, namely FamilyConcert, Jazz, JazzWorldMusic, Classical, Popular, RedSofa and WorldMusic. Similar to the day of the concert, the genre of the concert is thus used to calculate 14 variables. The 7 first variables, FamilyConcertCount, JazzCount, JazzWorldMusicCount, ClassicalCount, PopularCount, RedSofaCount and WorldMusicCount, are calculated at the customer level and represent the number of concerts, attended by the customer, of the respective genres. 7 other ratio-variables, FamilyConcertRatio, JazzRatio, JazzWorldMusicRatio, ClassicalRatio, PopularRatio, RedSofaRatio and WorldMusicRatio, are then created. These 28 variables are reported in Tables 5.2, together with their respective ranges.

At this point, a segmentation based on the variables of Table 5.1 of the 82082 customers of EventOrga is 
considered together with the 28 variables of Table 5.2 which are calculated for the 82082 customers, also based on six years of data. Referring to the theoretical section, a set $D$ of candidate variables and a prior segmentation $K$ of the data points of a set $N$ are created. The set $D$ gathers the 28 variables of Table 5.2, the partitioning $K$ is based on the 20 segments obtained in the previous section and the set $N$ represents the 82082 customers. Looking for a set of new variables can then be achieved by ranking the variables of the set $D$ conditionally to the clustering $K$. Since different techniques are available (the 7 techniques of Section 3 and the approach proposed in Section 4), multiple rankings of the candidate variables can be obtained as shown on Table 5.2 where the positions in the rankings of the 28 variables are reported for each of the 8 identified ranking approaches. A variable at the position 1 of the ranking obtained by applying a given strategy being the best candidate variable according to this respective strategy.

As can be seen on Table 5.2, the different ranking strategies are leading to different results. In order to select the one to adopt, some experiments are conducted based on the results of Table 5.2. The purpose of these experiments is to evaluate the different strategies by measuring the quality of the segments obtained when following these respective strategies. Each strategy is thus considered to build a series of partitions by iteratively adding new variables from the set of candidate variables to the set of original variables while following the order of the respective rankings. $224(8 \times 28)$ subsets of the candidate variables are thus formed, 186 of them being unique, leading to 186 executions of the clustering approach discussed in Section 2. The four evaluation metrics of Section 2 are then applied on the different clustering results in order to assess their quality. Since the absolute values of the different metrics are difficult to interpret, a relative comparison of the different strategies is to be preferred. These comparisons are reported on Figures 5(a), 5(b), 5(c) and 5(d) where the relative values of the respective metrics are reported for each of the 8 strategies. As an example of the reading of such a plot, Figure 5(a) allows to compare the 8 ranking strategies using the RMSSTD index introduced in Section 2. For each of the strategies, a blue line represents the RMSSTD values obtained after adding a number of candidate variables $\beta \in[1 . .28]$ (represented on the abscissa) following the given strategy. The solid black lines represent respectively the maximum and the minimum values of the RMSSTD of the 186 clustering output, such that the respective RMSSTD curves evolve between them. The dotted red line is a visual marker equidistant from the minimum and the maximum helping the analysis. This allows a visualization and a comparison of the different strategies using relative values, hence avoiding a difficult interpretation of the absolute values. As an example, comparing the PCA and the RandomForest rankings using the $\mathrm{CH}$ evaluation metric leads, referring to Figure 5(c), to the straightforward conclusion that the PCA strategy generates better clustering results on this database since a high value of $\mathrm{CH}$ is preferable. However, making the same comparison using the DB index, hence referring to Figure 5(c), is less straightforward since the curves are more similar.

In order to conclude on which strategy to adopt, one is thus looking for a strategy showing a superior behavior for the 4 different evaluation metrics (i.e. curves with higher values of RMSSTD, lower values of RS, higher values of $\mathrm{CH}$ and lower values of DB). Starting with the RMSSTD index, two strategies are showing relatively better performances (curves with higher values), namely the CS and the FCA. Looking closer on Figure 5(a), one could even say that the CS strategy is showing a relatively similar behavior to PCA until the first 20 new variables are added and performs slightly better for the 8 last variables until converging to the same point (which makes sense since the final clustering is similar for all strategies because all the candidate variables are added). Referring 


\begin{tabular}{|c|c|c|c|c|c|c|c|c|}
\hline & $\stackrel{\Re}{\tilde{U}}$ & 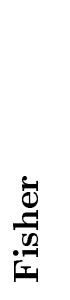 & 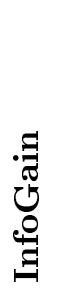 & 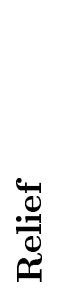 & 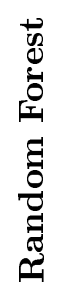 & $\underset{\widetilde{J}}{\mathbb{S}}$ & $\begin{array}{l}\overleftrightarrow{L} \\
0\end{array}$ & U \\
\hline FamilyConcertCount & 24 & 15 & 24 & 23 & 23 & 4 & 11 & 8 \\
\hline JazzCount & 26 & 23 & 26 & 26 & 15 & 20 & 14 & 11 \\
\hline JazzWorldMusicCount & 27 & 27 & 27 & 28 & 21 & 11 & 26 & 12 \\
\hline ClassicalCount & 1 & 1 & 1 & 18 & 18 & 2 & 1 & 1 \\
\hline PopularCount & 19 & 14 & 16 & 22 & 20 & 3 & 16 & 13 \\
\hline RedSofaCount & 23 & 16 & 25 & 25 & 11 & 24 & 9 & 6 \\
\hline WorldMusicCount & 25 & 19 & 22 & 24 & 9 & 6 & 13 & 14 \\
\hline FamilyConcertRatio & 20 & 21 & 19 & 10 & 8 & 16 & 17 & 23 \\
\hline JazzRatio & 22 & 17 & 23 & 13 & 27 & 27 & 22 & 9 \\
\hline JazzWorldMusicRatio & 28 & 28 & 28 & 27 & 22 & 8 & 28 & 15 \\
\hline ClassicalRatio & 2 & 6 & 2 & 1 & 17 & 12 & 10 & 19 \\
\hline PopularRatio & 12 & 8 & 9 & 3 & 12 & 28 & 12 & 16 \\
\hline RedSofaRatio & 17 & 26 & 20 & 19 & 6 & 25 & 27 & 17 \\
\hline WorldMusicRatio & 18 & 11 & 18 & 7 & 2 & 17 & 15 & 10 \\
\hline MondayCount & 21 & 10 & 21 & 6 & 24 & 19 & 8 & 20 \\
\hline TuesdayCount & 15 & 9 & 15 & 20 & 14 & 10 & 6 & 4 \\
\hline WednesdayCount & 16 & 7 & 17 & 21 & 25 & 13 & 7 & 18 \\
\hline ThursdayCount & 9 & 4 & 11 & 17 & 26 & 23 & 5 & 5 \\
\hline FridayCount & 7 & 2 & 8 & 14 & 16 & 1 & 2 & 2 \\
\hline SaturdayCount & 8 & 3 & 7 & 16 & 10 & 21 & 3 & 3 \\
\hline SundayCount & 13 & 5 & 12 & 15 & 13 & 26 & 4 & 7 \\
\hline MondayRatio & 14 & 18 & 14 & 12 & 4 & 9 & 20 & 21 \\
\hline TuesdayRatio & 10 & 13 & 10 & 9 & 5 & 7 & 24 & 22 \\
\hline WednesdayRatio & 11 & 25 & 13 & 11 & 28 & 15 & 23 & 24 \\
\hline ThursdayRatio & 5 & 20 & 6 & 8 & 7 & 5 & 21 & 26 \\
\hline FridayRatio & 4 & 12 & 4 & 2 & 3 & 14 & 19 & 25 \\
\hline SaturdayRatio & 3 & 22 & 3 & 5 & 1 & 22 & 25 & 27 \\
\hline SundayRatio & 6 & 24 & 5 & 4 & 19 & 18 & 18 & 28 \\
\hline
\end{tabular}

Table 3: Position of each of the 28 variables for each of the 8 ranking strategies. 


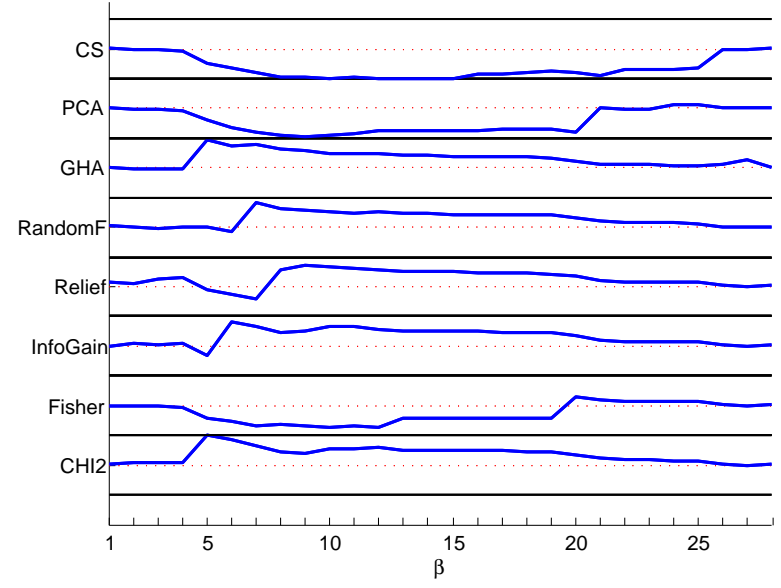

(a) $R M$

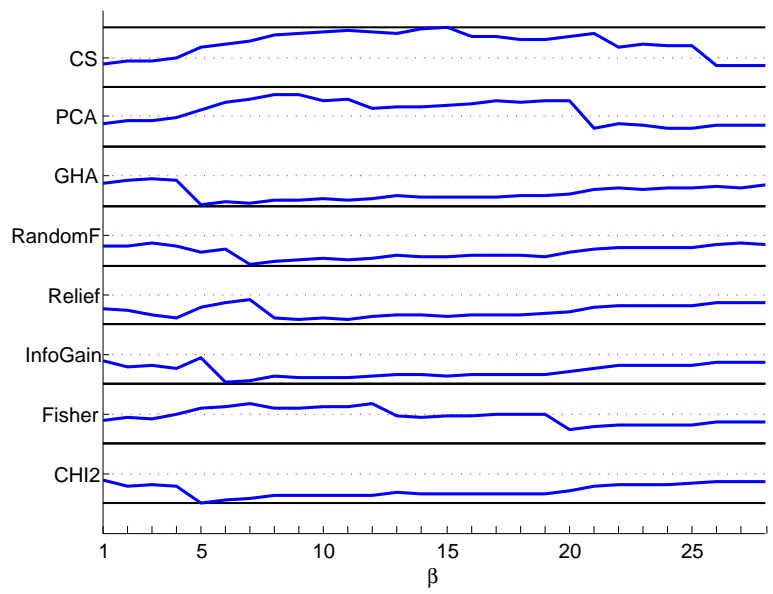

(c) $\mathrm{CH}$

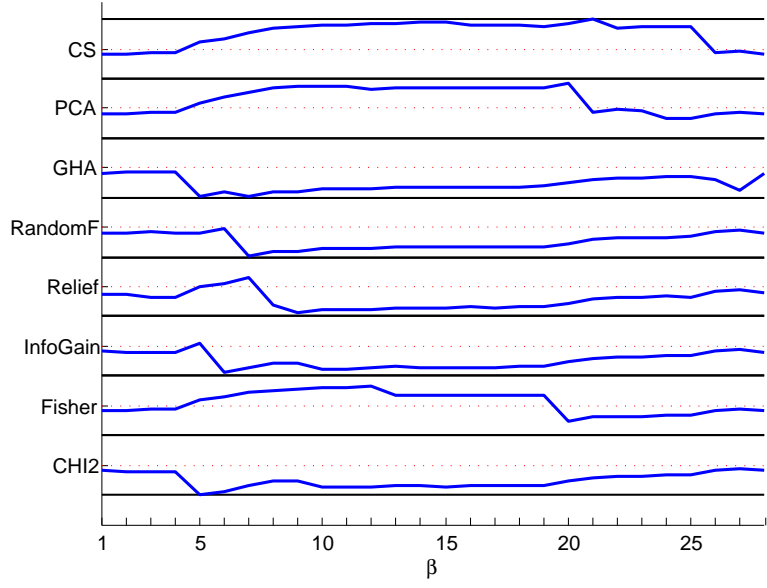

(b) $R S$

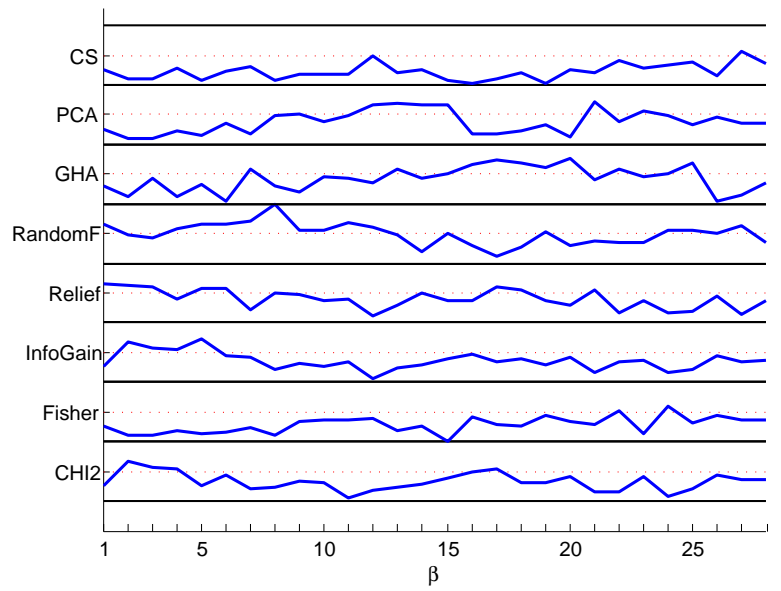

(d) $D B$

Figure 5: Relative values for the 4 evaluation metrics RMSSTD, RS, CH and DB for different values of $\beta$ considering the 8 strategies. 
to Figure 5(b), 3 strategies can be identified as performing better (higher RS values than the other strategies), namely the CS, PCA and Fisher Score strategies. As for the previous evaluation metric, a closer look tends to confirm that even if the curves are relatively similar when adding the first candidate variables, the CS strategy is performing equally or better when adding the last variables. Referring to Figure 5(c), the CS strategy clearly outperforms the other strategies, showing higher values of $\mathrm{CH}$ when adding new variables. Finally, when referring to the DB index of Figure 5(d), the CS and Fisher Score strategies seem to perform better than the other strategies, showing relatively lower values for the DB index. As a conclusion, the CS, PCA and the Fisher Score strategies seem to perform better on the data set at hand than the other 5 strategies. Looking closer and comparing these 3 strategies, the CS approach is showing similar or better results than the other strategies when analyzing the different evaluation metrics. Because of these results and the ease of calculation of the CS strategy, this approach is considered, for this case, as the strategy to adopt. Note that the experiments leading to the plots of Figure 5 are computationally expensive and are reported here in order to assess the proposed feature selection approaches by simulating the different possible outcomes. Other experiments with additional data sets may enrich this analysis but, given the business objective of this application, focusing on this data set will already allow us to make different decisions. For example, based on the selected strategy, the CS strategy, and referring to Table 5.2, it could be said that the ratios about the preferences for the different days are not relevant candidates to add to the original variables since they are ranked last according to the CS ranking. One could then decide to choose a set of $\beta$ new variables to add to the existing segmentation by adding to the original variables the $\beta$ variables ranked first when adopting this same strategy (using Table 5.2 for the rankings). Since the focus of this paper is on providing a ranking of the candidate variables and not on providing an optimal set of variables to add, the parameter $\beta$ is in this case an arbitrary parameter fixed by the user. The reason behind this choice is that proposing a framework for finding an optimal value for $\beta$ would be difficult to defend. It could be approached by considering the curve of a strategy for a given evaluation metric, as reported on Figure 5, and by trying to identify a value of $\beta$ maximizing this quality criterion. However, the arbitrary parameter would then become the evaluation metric to choose. For this reason, the different strategies are objectively assessed using four well-known evaluation techniques, leading to the identification of the best strategy for the data at hand, while keeping $\beta$ as the arbitrary parameter. The business can thus for example be interested in adding the 10 next best variables, such that $\beta$ is set to 10. Following the CS strategy, the 10 variables ClassicalCount, FridayCount, SaturdayCount, TuesdayCount, ThursdayCount, RedSofaCount, SundayCount, JazzRatio, FamilyConcertCount and WorldMusicRatio are thus added to the original variables in order to form a new segmentation taking into account the previous one while aiming at obtaining a better segmentation of the customers. After running the clustering approach of Section 2 on the new input, 20 clusters are obtained including the original variables and the 10 new candidate variables. The centers of these clusters and the number and percentage of customers belonging to each of them are reported in Figure 6, the interpretation of which being the same as for Figure 4.

\section{Conclusion}

In conclusion, this paper contributes to both research and business societies by proposing and applying an approach allowing to identify next relevant variables for segmentation by using feature selection techniques. From 


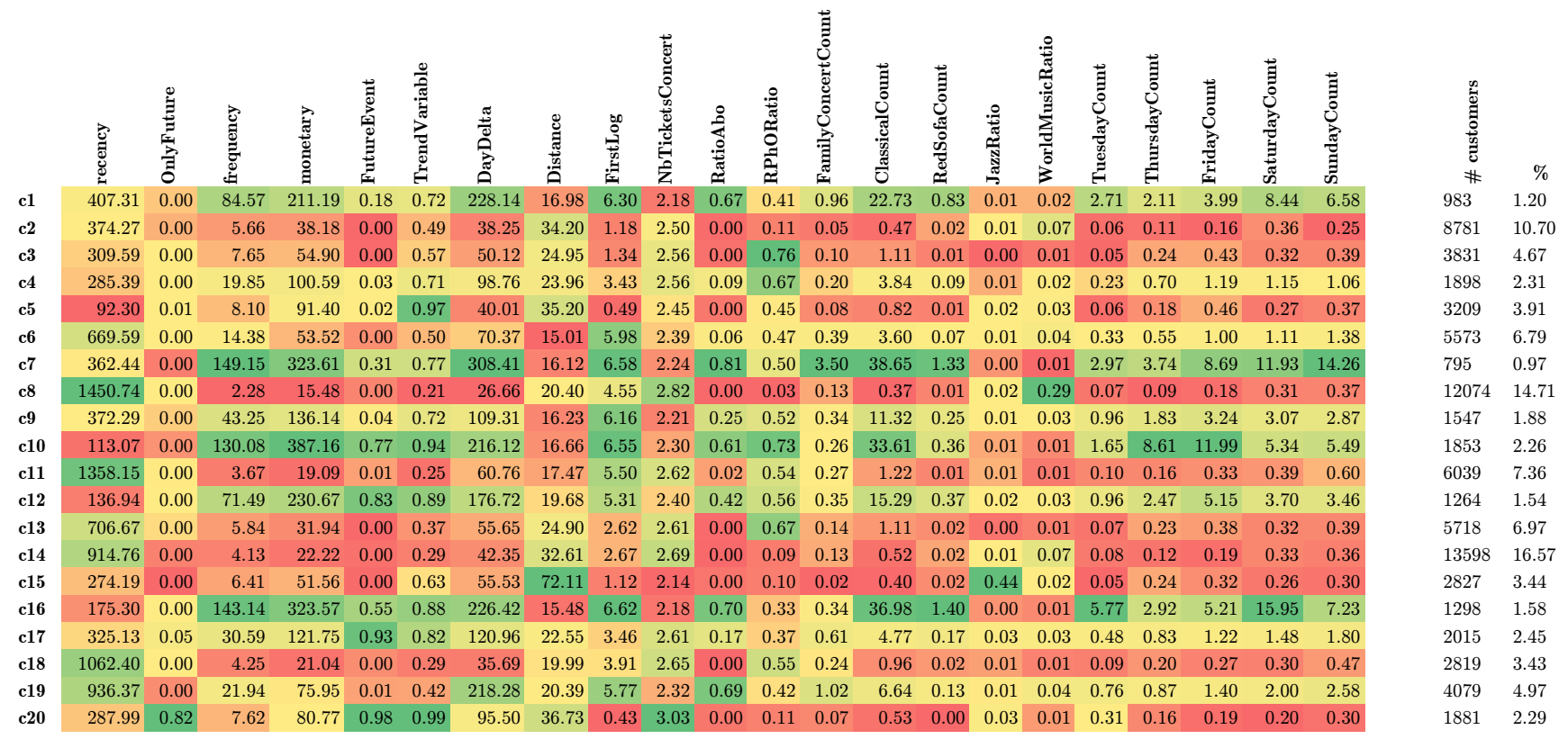

Figure 6: The centroids of the 20 clusters obtained after adding 10 of the candidate variables to the original variables.

a research point of view, a new problem is identified and formalized while discussing and unifying 7 candidate solutions issued from different domains. In addition, a new ranking approach is proposed based on fundamental criteria from the clustering discipline, adapted to provide a solution to the identified problem of ranking a new set of candidate variables conditionally to the structure of an existing segmentation with as objective to improve the quality of the resulting partition. In order to evaluate this quality, four evaluation metrics widely used and discussed in the literature are discussed and summarized. Experiments are then conducted in order to assess the different ranking strategies and are reported in detail. From a business perspective, a detailed real-life segmentation exercise is reported using a recent case from the concert industry while sharing insights collected during this application. After describing the different steps and output leading to the original segmentation of a customer base, the operational problem of how to update this segmentation by adding new variables is studied. The different techniques introduced in this paper are therefore combined in order to identify the best strategy to adopt given the problem and data set at hand. A ranking of a set of new variables is then obtained using the identified best strategy, allowing to discriminate irrelevant variables and to propose a new segmentation of the customer base enriched with a set of relevant new variables.

The main implication of applying this approach is that a new segmentation can be obtained conditionally to a previously obtained segmentation by adding new relevant variables. Practitioners should consider this approach if they face a situation in which a recent segmentation is already integrated and used in daily practices and new variables are available. This approach should then be preferred to a traditional re-clustering since the knowledge present in the first segmentation will be used to guide the new segmentation. In other words, since the current practices are based on the current segmentation, guiding the new segmentation using the existing knowledge will also guide the new practices by valuing existing ones, easing their acceptance by the business.

Finally, these experiments and associated results open new tracks for research since: 
- new ranking techniques could be developed or compared using the same setup,

- new evaluation metrics for clustering could be added,

- new questions could be answered as how to identify the optimal value for the parameter $\beta$ and

- similar experiments could be conducted using other data sets.

Note that the data, output and problems mentioned in this paper are based on a real case involving a leading concert organizer based in the Netherlands. We extend our gratitude to Mathijs Bouwman for his input as business expert, which significantly increased the value of this work.

\section{References}

Charu C. Aggarwal and Chandan K. Reddy, editors. Data Clustering: Algorithms and Applications. CRC Press, 2014 .

A. P. Azcarraga, M. H. Hsieh, S. L. Pan, and R. Setiono. Extracting salient dimensions for automatic SOM labeling. Systems, Man, and Cybernetics, Part C: Applications and Reviews, IEEE Transactions on, 35(4):595-600, 2005.

Bart Baesens. Analytics in a Big Data World: The Essential Guide to Data Science and its Applications. Wiley, 2014.

L. Breiman. Random forests. Machine Learning, 45(1):5-32, 2001.

Richard Colombo and Weina Jiang. A stochastic rfm model. Journal of Interactive Marketing, 13(3):2-12, 1999.

R.O. Duda, P.E. Hart, and D.G. Stork. Pattern Classification. John Wiley \& Sons, New York, 2 edition, 2001.

I. Guyon, S. Gunn, M. Nikravesh, and L. A. Zadeh. Feature extraction, foundations and applications. Springer, Berlin, 2006.

Donald E. Hebb. The Organization of Behavior. Wiley, New York, 1949.

Abdulkadir Hiziroglu. Soft computing applications in customer segmentation: State-of-art review and critique. Expert Systems with Applications, 40(16):6491 - 6507, 2013.

Teuvo Kohonen. Self-Organizing Maps. Springer, 1995.

I Kononenko. Estimating attributes: Analysis and extensions of relief. pages 171-182. Springer Verlag, 1994.

John A. Lee and Michel Verleysen. Nonlinear Dimensionality Reduction. Information Science and Statistics. Springer, Berlin, Heidelberg, 2007.

Philippe Louis, Alex Seret, and Bart Baesens. Financial efficiency and social impact of microfinance institutions using self-organizing maps. World Development, 46(0):197 - 210, 2013. 
K. Pearson. On the criterion that a given system of deviations from the probable in the case of a correlated system of variables is such that it can be reasonably supposed to have arisen from random sampling. Philosophical Magazine, 50(302):157-175, 1900.

Weber R. Nowatzke R. Peters, G. Dynamic rough clustering and its applications. Applied Soft Computing, 12(10): 3193-3207, 2012.

T. D. Sanger. Optimal unsupervised learning in a single-layer linear feedforward neural network. Neural Networks, 2(6):459-473, 1989.

Alex Seret, Thomas Verbraken, Versailles Sebastien, and Bart Baesens. A new som-based method for profile generation: Theory and an application in direct marketing. European Journal of Operational Research, 220, 2012.

Alex Seret, Seppe K.L.M. vanden Broucke, Bart Baesens, and Jan Vanthienen. A dynamic understanding of customer behavior processes based on clustering and sequence mining. Expert Systems with Applications, 41(10): 4648 - 4657, 2014a.

Alex Seret, Thomas Verbraken, and Bart Baesens. A new knowledge-based constrained clustering approach: Theory and application in direct marketing. Applied Soft Computing, 24(0):316 - 327, 2014b.

P.N. Tan, M. Steinbach, V. Kumar, et al. Introduction to Data Mining. Pearson Addison Wesley Boston, 2006.

Juha Vesanto and Esa Alhoniemi. Clustering of the self-organizing map. IEEE transactions on neural networks, 11 (3):586-600, 2000.

Kiri Wagstaff, Claire Cardie, Seth Rogers, and Stefan Schroedl. Constrained k-means clustering with background knowledge. In In ICML, pages 577-584. Morgan Kaufmann, 2001.

Yiming Yang and Jan O. Pedersen. A comparative study on feature selection in text categorization. pages 412-420. Morgan Kaufmann Publishers, 1997. 